\title{
ON LOCAL SMOOTH SOLUTIONS FOR THE VLASOV EQUATION WITH THE POTENTIAL OF INTERACTIONS $\pm r^{-2}$
}

\author{
PETER ZHIDKOV
}

Received 4 December 2003

\begin{abstract}
For the initial value problem for the Vlasov equation with the potential of interactions $\pm r^{-2}$, we prove the existence and uniqueness of a local solution with values in the Schwartz space $S$ of infinitely differentiable functions rapidly decaying at infinity.
\end{abstract}

2000 Mathematics Subject Classification: 35Q72, 82C05, 82C40.

1. Introduction, notation, and results. Vlasov equation and its various modifications are classical equations of physics. They appear in the mean-field approximations of the dynamics of a large number of interacting classical particles (molecules). Currently, there is a numerous literature devoted to its mathematical treatments. In particular, in [2, 5, 7, 12] a well-posedness for this equation supplied with initial data and its derivation from molecular dynamics are considered in the case when the potential of interactions between particles is smooth and bounded. In [1, 3, 6, 8, 10, 11, 13, 15], this equation is studied for the singular Coulomb potential $U(r)= \pm r^{-1}$ (in [6, 13], the Vlasov-Maxwell system and the two-dimensional Vlasov-Poisson system are considered, respectively). In [9], the local existence of smooth solutions in the case $U(r)= \pm r^{-2}$ is studied. We also mention paper [14] where a well-posedness of this equation supplied with a joint distribution of particles at two moments of time is proved.

In the present paper, we consider the problem

$$
\begin{gathered}
\frac{\partial}{\partial t} f+v \cdot \nabla_{x} f+\nabla_{v} f \cdot E(x, t)=0, \quad f=f(t, x, v), t \in \mathbb{R},(x, v) \in \mathbb{R}^{3} \times \mathbb{R}^{3}, \\
E(x, t)=\int_{\mathbb{R}^{3} \times \mathbb{R}^{3}} \nabla U(x-y) f(t, y, v) d y d v, \quad U(x)=\kappa|x|^{-2}, \kappa= \pm 1, \\
f(0, x, v)=f_{0}(x, v),
\end{gathered}
$$

where all quantities are real, $x, v \in \mathbb{R}^{3}, \kappa$ is a constant, $\nabla_{x}$ and $\nabla_{v}$ are the gradients in $x$ and $v$, respectively, $v \cdot \nabla_{x} f$ and $\nabla_{v} f \cdot E(x, t)$ are the scalar products in $\mathbb{R}^{3}$, and $f$ is an unknown function. For any fixed $t, f(t, x, v)$ regarded as a function of $(x, v)$ has the sense of a distribution function of particles in $(x, v) \in \mathbb{R}^{3} \times \mathbb{R}^{3}$. Therefore, the following requirements are natural:

$$
f(t, \cdot, \cdot) \geq 0, \quad \int_{\mathbb{R}^{3} \times \mathbb{R}^{3}} f(t, x, v) d x d v=1, \quad \forall t \in \mathbb{R} .
$$

Generally speaking, it is known that proving the existence of a solution for problem (1.1), (1.2), (1.3), and (1.4) is more difficult for a singular potential $U$ than for a more 
regular one. Also, although the Vlasov equation appeared for the first time with the Coulomb potential $U(r)= \pm r^{-1}$ for a description of plasma, it is well known that in statistical physics potentials with higher singularities occur, for example, the following one, the so-called Lennard-Jones potential, is known: $U(r)=A r^{-12}-B r^{-6}$. So, the author of the present paper believes that considerations of Vlasov equations for potentials with singularities of degrees higher than $r^{-1}$ make sense. Here we consider the case $U(r)= \pm r^{-2}$ proving the existence and uniqueness of a local smooth solution of the problem (1.1), (1.2), (1.3), and (1.4). This case is critical in a sense. A treatment of the problem in the case $U(r)=r^{-2-a}$ with $a>0$ is still left open. As for the case $U(r)=r^{-a}$ with $a \in(1,2)$, here the problem becomes simpler, and our methods still hold for it.

The existence and uniqueness of a smooth solution of problem (1.1), (1.2), (1.3), and (1.4) is proved in [9]. In fact, we prove a similar result by using another method that allows to treat the problem in a simpler and shorter way. We do not exploit the known method of characteristics but we use an approach known in the theory of nonlinear PDEs, too. Our results also hold for potentials of interaction of a more general kind $U(r)=\kappa r^{-2}+U_{1}(r)$, where $U_{1}(r)$ is a function continuously differentiable everywhere except maybe the point $r=0$ where it may have a singularity of order $|r|^{a-2}$ with $0<a<2$ and $U_{1}(r)$ must satisfy certain conditions of decay at infinity.

Now, we introduce some notation. Let

$S=\left\{g(x, v) \in C^{\infty}\left(\mathbb{R}^{3} \times \mathbb{R}^{3}\right): \forall k_{i}, m_{i}=0,1,2, \ldots, \sup _{(x, v) \in \mathbb{R}^{3} \times \mathbb{R}^{3}}|x|^{k_{1}}|v|^{k_{2}}\left|\frac{\partial^{m_{1}+m_{2}} g(x, v)}{\partial x^{m_{1}} \partial v^{m_{2}}}\right|<\infty\right\}$.

The linear space $S$ is equipped with a topology of open subsets becoming a complete topological space. This topology is generated by the system of seminorms

$$
\begin{aligned}
& p_{k, m}(g)=\left\{\int_{\mathbb{R}^{3} \times \mathbb{R}^{3}}\left(1+|x|^{2}\right)^{k_{1}}\left(1+|v|^{2}\right)^{k_{2}} \sum_{i=1}^{3}\left(\frac{\partial^{m} g(x, v)}{\partial x_{i}^{m}}\right)^{2} d x d v\right\}^{1 / 2}, \\
& q_{k, m}(g)=\left\{\int_{\mathbb{R}^{3} \times \mathbb{R}^{3}}\left(1+|x|^{2}\right)^{k_{1}}\left(1+|v|^{2}\right)^{k_{2}} \sum_{i=1}^{3}\left(\frac{\partial^{m} g(x, v)}{\partial v_{i}^{m}}\right)^{2} d x d v\right\}^{1 / 2},
\end{aligned}
$$

where $k=\left(k_{1}, k_{2}\right)$ with $k_{i}, m=0,1,2, \ldots$ By $C(I ; S)$, where $I \subset \mathbb{R}$ is an interval, we denote the linear space of all continuous functions $g: I \rightarrow S$ such that each seminorm $p_{k, m}(g(t))$ and $q_{k, m}(g(t))$ is bounded uniformly in $t \in I$.

Our main result here is the following.

THEOREM 1.1. Let $f_{0} \in S$ and satisfy (1.4). Then, there exists $T>0$ such that problem (1.1), (1.2), (1.3), and (1.4) has a unique local solution $f(t, x, v)$ satisfying $f(t, \cdot, \cdot), f_{t}^{\prime}(t, \cdot, \cdot)$ $\in C([-T, T] ; S)$.

REMARK 1.2. As is shown in [4], if $\kappa>0$, then problem (1.1), (1.2), (1.3), and (1.4) possesses solutions blowing up in finite intervals of time. So, in this case, generally speaking, solutions we consider can be not continuable onto the entire real line $t \in \mathbb{R}$. 
2. Proof of the theorem. Let $\omega(\cdot)$ be a nonnegative even $C^{\infty}$-function in $\mathbb{R}^{3}$ with a compact support satisfying $\int_{\mathbb{R}^{3}} \omega(x) d x=1$ and let $\omega_{n}(x)=n^{3} \omega(n x), n=1,2,3, \ldots$ Set $U_{n}(x)=\left(U \star \omega_{n}\right)(x)$, where the star means the convolution. Consider the following sequence of regularizations of problem (1.1), (1.2), (1.3), and (1.4):

$$
\begin{gathered}
f_{t}^{n}+v \cdot \nabla_{x} f^{n}+\nabla_{v} f^{n} \cdot E_{n}(x, t)=0, \quad f^{n}=f^{n}(t, x, v), \\
E_{n}(x, t)=\int_{\mathbb{R}^{3} \times \mathbb{R}^{3}} \nabla U_{n}(x-y) f^{n}(t, y, v) d y d v, \\
\left.f^{n}\right|_{t=0}=f_{0} \in S, \\
f^{n}(t, \cdot, \cdot) \geq 0, \quad \int_{\mathbb{R}^{3} \times \mathbb{R}^{3}} f^{n}(t, x, v) d x d v \equiv 1, \quad n=1,2,3, \ldots
\end{gathered}
$$

The following statement is a corollary of results in [2, 5, 7, 12].

Proposition 2.1. For each n, problem (2.1), (2.2), (2.3), and (2.4) has a unique global solution $f^{n}(t, x, v)$ that for any $t_{0}>0$ belongs to $C\left(\left(-t_{0}, t_{0}\right) ; S\right)$ together with $f_{t}^{n^{\prime}}(t, x, v)$.

Denote $\left(T_{n} g\right)(x)=\int_{\mathbb{R}^{3} \times \mathbb{R}^{3}} \nabla U_{n}(x-y) g(y, v) d y d v$, where $g \in S$. Note that

$$
\frac{\partial^{m}\left(T_{n} g\right)(x)}{\partial x_{1}^{m_{1}} \partial x_{2}^{m_{2}} \partial x_{3}^{m_{3}}} \equiv\left(T_{n}\left(\frac{\partial^{m} g(x, \cdot)}{\partial x_{1}^{m_{1}} \partial x_{2}^{m_{2}} \partial x_{3}^{m_{3}}}\right)\right)(x), \quad m=m_{1}+m_{2}+m_{3} \text {. }
$$

LEMmA 2.2. There exists $C>0$ such that $\left\|\left(T_{n} g\right)\right\|_{L_{2}\left(\mathbb{R}^{3}\right)} \leq C p_{(0,2), 0}(g)$ for all $g \in S$ and $n$.

Proof. As is well known, there exists $C_{1}>0$ such that

$$
\left\|\int_{\mathbb{R}^{3} \times \mathbb{R}^{3}} \nabla U(x-y) h(y) d y\right\|_{L_{2}\left(\mathbb{R}^{3}\right)} \leq C_{1}\|h\|_{L_{2}\left(\mathbb{R}^{3}\right)} \forall h \in S\left(\mathbb{R}^{3}\right) .
$$

Also,

$$
\int_{\mathbb{R}^{3} \times \mathbb{R}^{3}} \nabla U_{n}(x-y) g(y, v) d y d v=\int_{\mathbb{R}^{3} \times \mathbb{R}^{3}} \nabla U(x-y)\left(\omega_{n} \star_{x} g\right)(y, v) d y d v
$$

and $\left\|\omega_{n} \star h\right\|_{L_{2}\left(\mathbb{R}^{3}\right)} \leq\|h\|_{L_{2}\left(\mathbb{R}^{3}\right)}$ for all $h \in S\left(\mathbb{R}^{3}\right)$. Hence, we have, for $g \in S$,

$$
\begin{aligned}
& \left\|\int_{\mathbb{R}^{3} \times \mathbb{R}^{3}} \nabla U_{n}(x-y) g(y, v) d y d v\right\|_{L_{2}\left(\mathbb{R}^{3}\right)} \leq C_{1}\left\|\int_{\mathbb{R}^{3}} g(\cdot, v) d v\right\|_{L_{2}\left(\mathbb{R}^{3}\right)} \\
& \quad=C_{1}\left\|\int_{\mathbb{R}^{3}} \frac{\left(1+|v|^{2}\right) g(\cdot, v) d v}{\left(1+|v|^{2}\right)}\right\|_{L_{2}\left(\mathbb{R}^{3}\right)} \leq C\left\{\int_{\mathbb{R}^{3} \times \mathbb{R}^{3}}\left(1+|v|^{2}\right)^{2} g^{2}(x, v) d x d v\right\}^{1 / 2} \\
& =C p_{(0,2), 0}(g) .
\end{aligned}
$$

Let $[a]$ be the maximal integer not larger than a real $a$ and let an integer $m_{0}>0$ be such that each Sobolev space $H^{l+m_{0}}\left(\mathbb{R}^{3} \times \mathbb{R}^{3}\right)$ with a positive integer $l$ is embedded into $C^{l}\left(\mathbb{R}^{3} \times \mathbb{R}^{3}\right)$ (in fact, $m_{0}>3$ ). Let $m_{1}=2 m_{0}+2$. Everywhere $\bar{k}$ denotes $k_{1}+k_{2}$. In what 
follows, we exploit the following embeddings $(g \in S)$ :

$$
\begin{gathered}
\left\{\int_{\mathbb{R}^{3} \times \mathbb{R}^{3}} d x d v\left(1+|x|^{2}\right)^{k_{1}}\left(1+|v|^{2}\right)^{k_{2}}\left(\frac{\partial^{l} \boldsymbol{g}}{\partial x_{i}^{n} \partial v_{j}^{r}}\right)^{2}\right\}^{1 / 2} \\
\leq C\left[p_{k, 0}(g)+p_{k, s}(g)+q_{k, s}(g)\right], \\
p_{k, m}(g) \leq C\left[p_{(\bar{k}, 0), m}(g)+p_{(0, \bar{k}), m}(g)\right], \\
q_{k, m}(g) \leq C\left[q_{(\bar{k}, 0), m}(g)+q_{(0, \bar{k}), m}(g)\right], \quad m=0,1,2, \ldots, \\
\sup _{(x, v)}\left(1+|x|^{2}\right)^{k_{1} / 2}\left(1+|v|^{2}\right)^{k_{2} / 2}\left|\frac{\partial^{l} g(x, v)}{\partial x_{i}^{n} \partial v_{j}^{r}}\right| \leq C\left[p_{k, 0}(g)+p_{k, m+l}(g)+q_{k, m+l}(g)\right], \\
\sup _{x}\left\{\int_{\mathbb{R}^{3}}\left(1+|x|^{2}\right)^{k_{1}}\left(1+|v|^{2}\right)^{k_{2}}\left(\frac{\partial^{l} g(x, v)}{\partial x_{i}^{n} \partial v_{j}^{r}}\right)^{2} d v\right\}^{1 / 2} \\
\leq C\left[p_{k, 0}(g)+p_{k, l+m}(g)+q_{k, l+m}(g)\right],
\end{gathered}
$$

where $0 \leq l=n+r \leq s, i, j=1,2,3$, and $m=m_{0}, m_{0}+1, m_{0}+2, \ldots$. To prove them, consider the partition of $\mathbb{R}^{3} \times \mathbb{R}^{3}$ by cubes $K_{\alpha}$ defined by $r_{i}-1 \leq x_{i} \leq r_{i}, p_{j}-1 \leq v_{j} \leq p_{j}$, where $r_{i}, p_{j}$ run over all integers. Observe that for any integer $k_{1}, k_{2} \geq 0$ there exist $0<c<C$ such that

$$
c \leq \frac{\sup _{(x, v) \in K_{\alpha}}\left(1+|x|^{2}\right)^{k_{1}}\left(1+|v|^{2}\right)^{k_{2}}}{\inf _{(x, v) \in K_{\alpha}}\left(1+|x|^{2}\right)^{k_{1}}\left(1+|v|^{2}\right)^{k_{2}}} \leq C
$$

for all $\alpha$. Now, inequalities (2.9) follow by standard Sobolev embeddings applied to each cube $K_{\alpha}$ with further summing in $\alpha$.

LEMMA 2.3. There exist $T>0$ and $C>0$ such that

$$
p_{(0,2), 0}^{2}\left(f^{n}(t, \cdot, \cdot)\right)+p_{(0,2), m_{1}}^{2}\left(f^{n}(t, \cdot, \cdot)\right)+q_{(0,2), m_{1}}^{2}\left(f^{n}(t, \cdot, \cdot)\right) \leq C
$$

for all $t \in[-T, T]$ and all $n=1,2,3, \ldots$

Proof. We derive our estimates only for $t>0$ because the case $t<0$ can be treated by analogy. Integrating by parts in $v$ using the independence of $E_{n}(x, t)$ of $v$, estimating $E_{n}(x, t)$ by $p_{(0,2), 0}\left(f^{n}(t, \cdot, \cdot)\right)+p_{(0,2), m_{1}}\left(f^{n}(t, \cdot, \cdot)\right)$ according to the Sobolev embedding of $H^{m_{1}}\left(\mathbb{R}^{3}\right)$ into $C\left(\mathbb{R}^{3}\right)$, Lemma 2.2 , and the fact that $\partial^{m_{1}} E_{n}(x, t) / \partial x^{m_{1}}=$ $T_{n}\left(\partial^{m_{1}} f^{n}(t, x, v) / \partial x^{m_{1}}\right)$, and applying Hölder's inequality, we have, from (2.1),

$$
\begin{aligned}
\frac{1}{2} \frac{d}{d t} & p_{(0,2), 0}^{2}\left(f^{n}(t, \cdot, \cdot)\right) \\
& =-\int_{\mathbb{R}^{3} \times \mathbb{R}^{3}}\left(1+|v|^{2}\right)^{2} f^{n}(t, x, v) f_{v}^{n^{\prime}}(t, x, v) \cdot E_{n}(x, t) d x d v \\
& =2 \int_{\mathbb{R}^{3} \times \mathbb{R}^{3}}\left(1+|v|^{2}\right) v \cdot E_{n}(x, t)\left(f^{n}(t, x, v)\right)^{2} d x d v \\
& \leq C p_{(0,2), 0}^{2}\left(f^{n}(t, \cdot, \cdot)\right)\left[p_{(0,2), 0}\left(f^{n}(t, \cdot, \cdot)\right)+p_{(0,2), m_{1}}\left(f^{n}(t, \cdot, \cdot)\right)\right] .
\end{aligned}
$$


Consider

$$
\begin{aligned}
\frac{1}{2} \frac{d}{d t} p_{(0,2), m_{1}}^{2}\left(f^{n}(t, \cdot, \cdot)\right)= & -\int_{\mathbb{R}^{3} \times \mathbb{R}^{3}} d x d v\left(1+|v|^{2}\right)^{2} \sum_{i, j=1}^{3} f_{x_{i}}^{n\left(m_{1}\right)}(t, x, v) \\
& \times \sum_{l=0}^{m_{1}}\left(\begin{array}{c}
m_{1} \\
l
\end{array}\right) \frac{\partial^{l+1} f^{n}(t, x, v)}{\partial v_{j} \partial x_{i}^{l}} \frac{\partial^{m_{1}-l}}{\partial x_{i}^{m_{1}-l}} E_{n, j}(x, t) .
\end{aligned}
$$

To estimate the term with the $\left(m_{1}+1\right)$ st derivative of $f^{n}$, we integrate by parts in $v$ and use the fact that $E_{n}(x, t)$ does not depend on $v$. Also, as above, we estimate the uniform norm of $E_{n}(x, t)$ by $p_{(0,2), 0}\left(f^{n}(t, \cdot, \cdot)\right)+p_{(0,2), m_{1}}\left(f^{n}(t, \cdot, \cdot)\right)$ and apply Hölder's inequality. To estimate all other terms in the right-hand side of equation (2.13), observe that the order of one of the derivatives in $\left(1+|v|^{2}\right)\left(\partial^{l+1} f^{n}(t, x, v) / \partial v_{j} \partial x_{i}^{l}\right)$ and $\left(\partial^{m_{1}-l} / \partial x_{i}^{m_{1}-l}\right) E_{n, j}(x, t)$ is not larger than $m_{0}+1$ and therefore, either one has

$$
\left\|\frac{\partial^{m_{1}-l} E_{n, j}(x, t)}{\partial x_{i}^{m_{1}-l}}\right\|_{C\left(\mathbb{R}^{3}\right)} \leq C\left[p_{(0,2), 0}\left(f^{n}(t, \cdot, \cdot)\right)+p_{(0,2), m_{1}}\left(f^{n}(t, \cdot, \cdot)\right)\right]
$$

or the estimate in the fourth string of (2.9) takes place; in addition, we estimate the $L_{2}$-norm of the other cofactor with the derivative of order larger than $m_{0}+1$ by the same quantity corresponding to it. So, applying Hölder's inequality, we arrive at the estimate

$$
\begin{aligned}
& \frac{1}{2} \frac{d}{d t} p_{(0,2), m_{1}}^{2}\left(f^{n}(t, \cdot, \cdot)\right) \\
& \leq C p_{(0,2), m_{1}}^{2}\left(f^{n}(t, \cdot, \cdot)\right)\left[p_{(0,2), 0}\left(f^{n}(t, \cdot, \cdot)\right)+p_{(0,2), m_{1}}\left(f^{n}(t, \cdot, \cdot)\right)\right] \\
&+ \mid \int_{\mathbb{R}^{3} \times \mathbb{R}^{3}} d x d v\left(\sum_{l=0}^{m_{0}+1}+\sum_{l=m_{0}+2}^{m_{1}-1}\right) \sum_{i, j=1}^{3}\left(1+|v|^{2}\right)^{2}\left(\begin{array}{c}
m_{1} \\
l
\end{array}\right) \\
& \times f_{x_{i}}^{n\left(m_{1}\right)}(t, x, v) \frac{\partial^{l+1} f^{n}(t, x, v)}{\partial v_{j} \partial x_{i}^{l}} \frac{\partial^{m_{1}-l} E_{n, j}(x, t)}{\partial x_{i}^{m_{1}-l}} \mid \\
& \leq C p_{(0,2), m_{1}}\left(f^{n}(t, \cdot, \cdot)\right) \\
& \times\left[p_{(0,2), 0}\left(f^{n}(t, \cdot, \cdot)\right)+p_{(0,2), m_{1}}\left(f^{n}(t, \cdot, \cdot)\right)\right] \\
& \times\left[p_{(0,2), 0}\left(f^{n}(t, \cdot, \cdot)\right)+p_{(0,2), m_{1}}\left(f^{n}(t, \cdot, \cdot)\right)+q_{(0,2), m_{1}}\left(f^{n}(t, \cdot, \cdot)\right)\right] .
\end{aligned}
$$

Finally, we deduce by complete analogy

$$
\begin{array}{rl}
\frac{1}{2} \frac{d}{d t} q_{(0,2), m_{1}}^{2}\left(f^{n}(t, \cdot, \cdot)\right) \\
\leq C & C\left[p_{(0,2), 0}^{2}\left(f^{n}(t, \cdot, \cdot)\right)+p_{(0,2), m_{1}}^{2}\left(f^{n}(t, \cdot, \cdot)\right)+q_{(0,2), m_{1}}^{2}\left(f^{n}(t, \cdot, \cdot)\right)\right] \\
& +\left|\int_{\mathbb{R}^{3} \times \mathbb{R}^{3}}\left(1+|v|^{2}\right)^{2} \sum_{i, j=1}^{3} \partial_{v_{j}} f_{v_{i}}^{n\left(m_{1}\right)}(t, x, v) f_{v_{i}}^{n\left(m_{1}\right)}(t, x, v) E_{n, j}(x, t) d x d v\right| \\
\leq & C\left[p_{(0,2), 0}^{2}\left(f^{n}(t, \cdot, \cdot)\right)+p_{(0,2), m_{1}}^{2}\left(f^{n}(t, \cdot, \cdot)\right)+q_{(0,2), m_{1}}^{2}\left(f^{n}(t, \cdot, \cdot)\right)\right] \\
& \times\left[p_{(0,2), 0}\left(f^{n}(t, \cdot, \cdot)\right)+p_{(0,2), m_{1}}\left(f^{n}(t, \cdot, \cdot)\right)+1\right] .
\end{array}
$$


Now, in view of (2.12), (2.13), (2.14), (2.15), and (2.16), denoting $A=p_{(0,2), 0}^{2}\left(f^{n}\right)+$ $p_{(0,2), m_{1}}^{2}\left(f^{n}\right)$ and $B=p_{(0,2), 0}^{2}\left(f^{n}\right)+q_{(0,2), m_{1}}^{2}\left(f^{n}\right)$, where $p_{(0,2), 0}\left(f^{n}\right) \equiv q_{(0,2), 0}\left(f^{n}\right)$, we obtain

$$
\begin{aligned}
& \dot{A}(t) \leq C_{1}(1+A(t)+B(t))^{3 / 2}, \\
& \dot{B}(t) \leq C_{2}(1+A(t)+B(t))^{3 / 2},
\end{aligned}
$$

and our claim is proved.

LEMMA 2.4. There exist $C_{k}>0$ such that $p_{k, m_{1}}\left(f^{n}(t, \cdot, \cdot)\right)+q_{k, m_{1}}\left(f^{n}(t, \cdot, \cdot)\right)+$ $p_{k, 0}\left(f^{n}\left(t, \cdot,^{\cdot}\right)\right) \leq C_{k}$ for all $t \in[-T, T]$ and all $k_{1}, k_{2}, n=1,2,3, \ldots$

Proof. Again, we consider only the case $t>0$. We have from (2.1)

$$
\begin{aligned}
\frac{1}{2} \frac{d}{d t} p_{(k, 0), m_{1}}^{2}\left(f^{n}(t, \cdot, \cdot)\right) & \\
\leq & C k\left[p_{(k, 0), m_{1}}^{2}\left(f^{n}(t, \cdot, \cdot)\right)+p_{(0, k), m_{1}}^{2}\left(f^{n}(t, \cdot, \cdot)\right)\right] \\
+ & \mid \int_{\mathbb{R}^{3} \times \mathbb{R}^{3}}\left(1+|x|^{2}\right)^{k} \times \sum_{i, j=1}^{3} f_{x_{i}}^{n\left(m_{1}\right)}(t, \cdot, \cdot)\left(\sum_{l=0}^{m_{0}+1}+\sum_{l=m_{0}+2}^{m_{1}-1}\right)\left(\begin{array}{c}
m_{1} \\
l
\end{array}\right) \frac{\partial^{l+1} f^{n}(t, \cdot, \cdot)}{\partial v_{j} \partial x_{i}^{l}} \\
& \times \frac{\partial^{m_{1}-l} E_{n, j}(x, t)}{\partial x_{i}^{m_{1}-l}} d x d v \mid .
\end{aligned}
$$

As earlier, we apply the embeddings (2.9) and Hölder's inequality and estimate the uniform norm of those of two expressions $\left(1+|x|^{2}\right)^{k / 2}\left(\partial^{l+1} f^{n}(t, x, v) / \partial v_{j} \partial x_{i}^{l}\right)$ and $\left(\partial^{m_{1}-l} E_{n, j}(x, t) / \partial x_{i}^{m_{1}-l}\right)$, the order of the derivative in which is not larger than $m_{0}+1$, by $C\left[p_{(k, 0), 0}\left(f^{n}(t, \cdot, \cdot)\right)+p_{(k, 0), m_{1}}\left(f^{n}(t, \cdot, \cdot)\right)+q_{(k, 0), m_{1}}\left(f^{n}(t, \cdot)\right)\right]$ or $C\left[p_{(0,2), 0}\left(f^{n}(t, \cdot, \cdot)\right)\right.$ $\left.+p_{(0,2), m_{1}}\left(f^{n}(t, \cdot, \cdot)\right)\right]$, respectively. In this way, applying (2.9), we obtain as when proving Lemma 2.3

$$
\begin{aligned}
& \frac{1}{2} \frac{d}{d t} p_{(k, 0), m_{1}}^{2}\left(f^{n}(t, \cdot, \cdot)\right) \\
& \leq C k\left[p_{(k, 0), m_{1}}^{2}\left(f^{n}(t, \cdot, \cdot)\right)+p_{(0, k), m_{1}}^{2}\left(f^{n}(t, \cdot, \cdot)\right)\right] \\
& +C p_{(k, 0), m_{1}}\left(f^{n}(t, \cdot, \cdot)\right)\left[p_{(k, 0), 0}\left(f^{n}(t, \cdot, \cdot)\right)+p_{(k, 0), m_{1}}\left(f^{n}(t, \cdot, \cdot)\right)\right. \\
& \left.+q_{(k, 0), m_{1}}\left(f^{n}(t, \cdot, \cdot)\right)\right] \\
& \times\left[p_{(0,2), 0}\left(f^{n}(t, \cdot, \cdot)\right)+p_{(0,2), m_{1}}\left(f^{n}(t, \cdot, \cdot)\right)\right] .
\end{aligned}
$$


By the complete analogy,

$$
\begin{aligned}
& \frac{1}{2} \frac{d}{d t} p_{(0, k), m_{1}}^{2}\left(f^{n}(t, \cdot, \cdot)\right) \\
& \leq C_{k}^{1} p_{(0, k), m_{1}}\left(f^{n}(t, \cdot, \cdot)\right)\left[p_{(0, k), 0}\left(f^{n}(t, \cdot, \cdot)\right)+p_{(0, k), m_{1}}\left(f^{n}(t, \cdot, \cdot)\right)\right. \\
& \left.+q_{(0, k), m_{1}}\left(f^{n}(t, \cdot, \cdot)\right)\right] \\
& \times\left[p_{(0,2), 0}\left(f^{n}(t, \cdot, \cdot)\right)+p_{(0,2), m_{1}}\left(f^{n}(t, \cdot, \cdot)\right)\right], \\
& \frac{1}{2} \frac{d}{d t} q_{(k, 0), m_{1}}^{2}\left(f^{n}(t, \cdot, \cdot)\right) \leq C_{k}^{2}\left[p_{(k, 0), 0}^{2}\left(f^{n}(t, \cdot, \cdot)\right)+p_{(0, k), 0}^{2}\left(f^{n}(t, \cdot, \cdot)\right)\right. \\
& \left.+q_{(k, 0), m_{1}}^{2}\left(f^{n}(t, \cdot, \cdot)\right)+q_{(0, k), m_{1}}^{2}\left(f^{n}(t, \cdot, \cdot)\right)\right], \\
& \frac{1}{2} \frac{d}{d t} q_{(0, k), m_{1}}^{2}\left(f^{n}(t, \cdot, \cdot)\right) \\
& \leq C_{k}^{3}\left[p_{(0, k), 0}^{2}\left(f^{n}(t, \cdot, \cdot)\right)+p_{(0, k), m_{1}}^{2}\left(f^{n}(t, \cdot, \cdot)\right)+q_{(0, k), m_{1}}^{2}\left(f^{n}(t, \cdot, \cdot)\right)\right] \\
& +C_{k}^{3} q_{(0, k), m_{1}}^{2}\left(f^{n}(t, \cdot, \cdot)\right)\left[p_{(0,2), 0}\left(f^{n}(t, \cdot, \cdot)\right)+p_{(0,2), m_{1}}\left(f^{n}(t, \cdot, \cdot)\right)\right], \\
& \frac{1}{2} \frac{d}{d t} p_{k, 0}^{2}\left(f^{n}(t, \cdot, \cdot)\right) \leq C_{k}^{4}\left[p_{(\bar{k}, 0), 0}^{2}\left(f^{n}(t, \cdot, \cdot)\right)+p_{(0, \bar{k}), 0}^{2}\left(f^{n}(t, \cdot, \cdot)\right)\right] \\
& \times\left[p_{(0,2), 0}\left(f^{n}(t, \cdot, \cdot)\right)+p_{(0,2), m_{1}}\left(f^{n}(t, \cdot, \cdot)\right)+1\right] .
\end{aligned}
$$

Now, in view of (2.18), (2.19), (2.20), (2.21), (2.22), (2.23), Lemma 2.3, and the embeddings (2.9), our result follows.

LEMmA 2.5. There exist $C_{k, m}>0$ such that $p_{k, m}\left(f^{n}(t, \cdot, \cdot)\right)+q_{k, m}\left(f^{n}(t, \cdot, \cdot)\right) \leq C_{k, m}$ for all $n, k, m$ and $t \in[-T, T]$.

Proof. Again, we establish all our estimates only for $t>0$. Let $m \geq 2 m_{0}+3$. It follows from (2.1) that

$$
\begin{aligned}
\frac{1}{2} \frac{d}{d t} p_{k, m}^{2} & \left(f^{n}(t, \cdot, \cdot)\right) \\
\leq C & \bar{k}\left[p_{(\bar{k}, 0), 0}^{2}\left(f^{n}(t, \cdot, \cdot)\right)+p_{(0, \bar{k}), 0}^{2}\left(f^{n}(t, \cdot, \cdot)\right)+p_{(\bar{k}, 0), m}^{2}\left(f^{n}(t, \cdot, \cdot)\right)\right. \\
& \left.+p_{(0, \bar{k}), m}^{2}\left(f^{n}(t, \cdot, \cdot)\right)\right] \\
+ & \int_{\mathbb{R}^{3} \times \mathbb{R}^{3}}\left(1+|x|^{2}\right)^{k_{1}}\left(1+|v|^{2}\right)^{k_{2}} \sum_{i, j=1}^{3} f_{x_{i}}^{n(m)}(t, x, v) \\
\times & {\left[\frac{\partial^{m+1} f^{n}}{\partial v_{j} \partial x_{i}^{m}} E_{n, j}(x, t)+\left(\sum_{l=0}^{[m / 2]}+\sum_{l=[m / 2]+1}^{m-1}\right)\right.} \\
& \left.\quad \times\left(\begin{array}{c}
m \\
l
\end{array}\right) \frac{\partial^{l+1} f^{n}(t, x, v)}{\partial v_{j} \partial x_{i}^{l}} \frac{\partial^{m-l} E_{n, j}(x, t)}{\partial x_{i}^{m-l}} d x d v\right] .
\end{aligned}
$$

The first term in the integrand in the right-hand side of this relation can be estimated as when proving Lemma 2.3: we integrate by parts in $v$ and apply the estimate

$$
\left\|E_{n, j}(\cdot, t)\right\|_{C\left(\mathbb{R}^{3}\right)} \leq C\left[p_{(0,2), 0}\left(f^{n}(t, \cdot, \cdot)\right)+p_{(0,2), m_{0}}\left(f^{n}(t, \cdot, \cdot)\right)\right] .
$$


As for the other terms in the integrand in (2.24), again, the order of one of two derivatives in $\left(1+|x|^{2}\right)^{k_{1} / 2}\left(1+|v|^{2}\right)^{k_{2} / 2}\left(\partial^{l+1} f^{n}(t, x, v) / \partial v_{j} \partial x_{i}^{l}\right)$ and $\left(\partial^{m-l} E_{n, j}(x, t) / \partial x_{i}^{m-l}\right)$ is not larger than $[m / 2]+1$ and so, the uniform norm of the corresponding expression can be estimated by $C\left[p_{k, 0}\left(f^{n}(t, \cdot, \cdot)\right)+p_{k, m-1}\left(f^{n}(t, \cdot, \cdot)\right)+q_{k, m-1}\left(f^{n}(t, \cdot, \cdot)\right)\right]$ or $C\left[p_{(0,2), 0}\left(f^{n}(t, \cdot, \cdot)\right)+p_{(0,2), m-1}\left(f^{n}(t, \cdot, \cdot)\right)\right]$, respectively; the $L_{2}$-norm of the other cofactor can be estimated in a similar way. So, as earlier, applying Hölder's inequality, we arrive at the estimate

$$
\begin{aligned}
& \frac{1}{2} \frac{d}{d t} p_{k, m}^{2}\left(f^{n}(t)\right) \\
& \leq C_{1}\left[p_{(\bar{k}, 0), 0}^{2}\left(f^{n}(t, \cdot, \cdot)\right)+p_{(0, \bar{k}), 0}^{2}\left(f^{n}(t, \cdot, \cdot)\right)+p_{(\bar{k}, 0), m}^{2}\left(f^{n}(t, \cdot, \cdot)\right)\right. \\
&\left.\quad+p_{(0, \bar{k}), m}^{2}\left(f^{n}(t, \cdot, \cdot)\right)\right] \\
&+C_{2} p_{k, m}^{2}\left(f^{n}(t, \cdot, \cdot)\right)\left[p_{(0,2), m_{0}}\left(f^{n}(t, \cdot, \cdot)\right)+p_{(0,2), 0}\left(f^{n}(t, \cdot, \cdot)\right)\right] \\
&+C_{2} p_{k, m}\left(f^{n}(t, \cdot, \cdot)\right)\left[p_{k, 0}\left(f^{n}(t, \cdot, \cdot)\right)+p_{k, m-1}\left(f^{n}(t, \cdot, \cdot)\right)+q_{k, m-1}\left(f^{n}(t, \cdot, \cdot)\right)\right] \\
& \times\left[p_{(0,2), 0}\left(f^{n}(t, \cdot, \cdot)\right)+p_{(0,2), m}\left(f^{n}(t, \cdot, \cdot)\right)\right] \\
&+ C_{2} p_{k, m}\left(f^{n}(t, \cdot, \cdot)\right)\left[p_{k, 0}\left(f^{n}(t, \cdot, \cdot)\right)+p_{k, m}\left(f^{n}(t, \cdot, \cdot)\right)+q_{k, m}\left(f^{n}(t, \cdot, \cdot)\right)\right] \\
& \times\left[p_{(0,2), 0}\left(f^{n}(t, \cdot, \cdot)\right)+p_{(0,2), m-1}\left(f^{n}(t, \cdot, \cdot)\right)\right] .
\end{aligned}
$$

By complete analogy

$$
\begin{aligned}
\frac{1}{2} \frac{d}{d t} q_{k, m}^{2} & \left(f^{n}(t, \cdot, \cdot)\right) \\
\leq & C\left[p_{(\bar{k}, 0), 0}^{2}\left(f^{n}(t, \cdot, \cdot)\right)+p_{(0, \bar{k}), 0}^{2}\left(f^{n}(t, \cdot, \cdot)\right)+q_{(\bar{k}, 0), m}^{2}\left(f^{n}(t, \cdot, \cdot)\right)\right. \\
& \left.\quad+q_{(0, \bar{k}), m}^{2}\left(f^{n}(t, \cdot, \cdot)\right)\right] \\
+ & C\left[q_{(\bar{k}, 0), m}^{2}\left(f^{n}(t, \cdot, \cdot)\right)+q_{(0, \bar{k}), m}^{2}\left(f^{n}(t, \cdot, \cdot)\right)\right] \\
& \times\left[p_{(0,2), 0}\left(f^{n}(t, \cdot, \cdot)\right)+p_{(0,2), m_{0}}\left(f^{n}(t, \cdot, \cdot)\right)\right] .
\end{aligned}
$$

Now, the statement of our lemma follows from (2.26), (2.27), and Lemmas 2.3 and 2.4 by induction.

In view of Lemmas 2.2, 2.3, 2.4, and 2.5 and (2.1), the sequences $\left\{f^{n}\right\}$ and $\left\{d f^{n} / d t\right\}$ are relatively compact in $C([-T, T] ; S)$. Without loss of generality, we can accept that these sequences converge and let $f(t, x, v)$ and $f_{1}(t, x, v)$ be their limit points in this sense. Clearly, $f_{t}^{\prime}(t, x, v) \equiv f_{1}(t, x, v)$ and $f$ satisfies problem (1.1), (1.2), (1.3), and (1.4). Now, we prove the uniqueness of this solution. Assume the existence of two solutions $f_{1}$ and $f_{2}$ of the above class and set $f=f_{1}-f_{2}$. As earlier, we establish our estimates only for $t>0$. One can obtain, as when proving Lemmas 2.3 and 2.4,

$$
\frac{d}{d t} p_{(0,2), 0}^{2}(f(t, \cdot, \cdot)) \leq C p_{(0,2), 0}^{2}(f(t, \cdot, \cdot)),
$$

therefore $f(t, x, v) \equiv 0$ for all $t \in[0, T]$, which completes our proof of the theorem. 


\section{REFERENCES}

[1] A. A. Arsen'ev, Global existence of a weak solution of Vlasov's system of equations, Comput. Math. Math. Phys. 15 (1975), 136-147.

[2] _ Lectures on Kinetic Equations, Nauka, Moscow, 1992.

[3] J. Batt, Global symmetric solutions of the initial value problem of stellar dynamics, J. Differential Equations 25 (1977), no. 3, 342-364.

[4] A. V. Bobylev, P. Dukes, R. Illner, and H. D. Victory, On Vlasov-Manev equations. I. Foundations, properties, and nonglobal existence, J. Statist. Phys. 88 (1997), no. 3-4, 885911.

[5] W. Braun and K. Hepp, The Vlasov dynamics and its fluctuations in the $1 / N$ limit of interacting classical particles, Comm. Math. Phys. 56 (1977), no. 2, 101-113.

[6] R. J. DiPerna and P.-L. Lions, Global weak solutions of Vlasov-Maxwell systems, Comm. Pure Appl. Math. 42 (1989), no. 6, 729-757.

[7] R. L. Dobrušin, Vlasov equations, Funktsional. Anal. i Prilozhen. 13 (1979), no. 2, 48-58 (Russian).

[8] E. Horst, On the asymptotic growth of the solutions of the Vlasov-Poisson system, Math. Methods Appl. Sci. 16 (1993), no. 2, 75-86.

[9] R. Illner, H. D. Victory, P. Dukes, and A. V. Bobylev, On Vlasov-Manev equations. II. Local existence and uniqueness, J. Statist. Phys. 91 (1998), no. 3-4, 625-654.

[10] P.-L. Lions and B. Perthame, Propagation of moments and regularity for the 3-dimensional Vlasov-Poisson system, Invent. Math. 105 (1991), no. 2, 415-430.

[11] J. Schaeffer, Global existence of smooth solutions to the Vlasov-Poisson system in three dimensions, Comm. Partial Differential Equations 16 (1991), no. 8-9, 1313-1335.

[12] H. Spohn, Large Scale Dynamics of Interacting Particles, Springer-Verlag, Berlin, 1991.

[13] S. Ukai and T. Okabe, On classical solutions in the large in time of two-dimensional Vlasov's equation, Osaka J. Math. 15 (1978), no. 2, 245-261.

[14] P. E. Zhidkov, On a problem with two-time data for the Vlasov equation, Nonlinear Anal. 31 (1998), no. 5-6, 537-547.

[15]___ On global solutions for the Vlasov-Poisson system, Electron. J. Differential Equations 2004 (2004), no. 58, 1-11.

Peter Zhidkov: Bogoliubov Laboratory of Theoretical Physics, Joint Institute for Nuclear Research, 141980 Dubna, Moscow region, Russia

E-mail address: zhidkov@thsun1.jinr.ru 


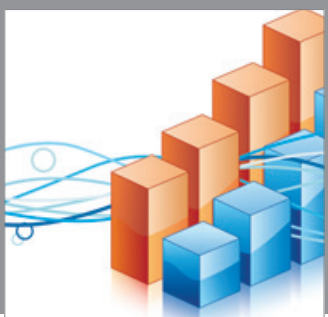

Advances in

Operations Research

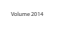

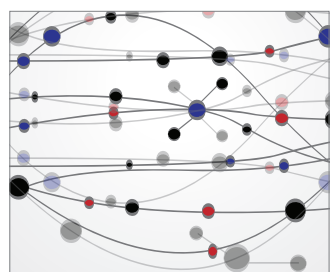

\section{The Scientific} World Journal
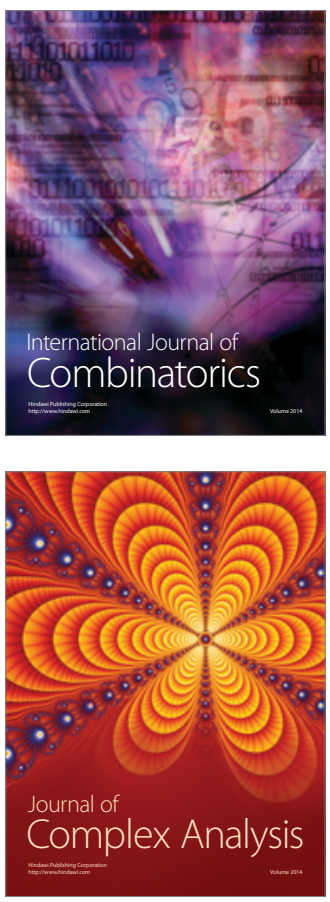

International Journal of

Mathematics and

Mathematical

Sciences
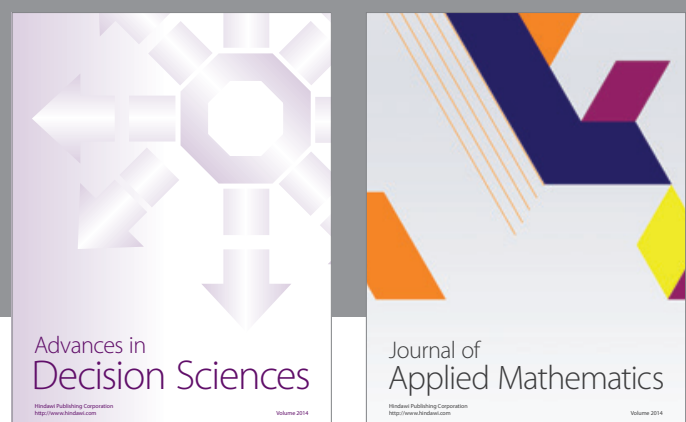

Journal of

Applied Mathematics
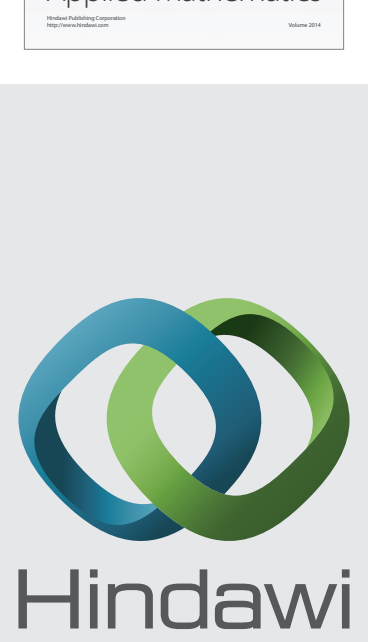

Submit your manuscripts at http://www.hindawi.com
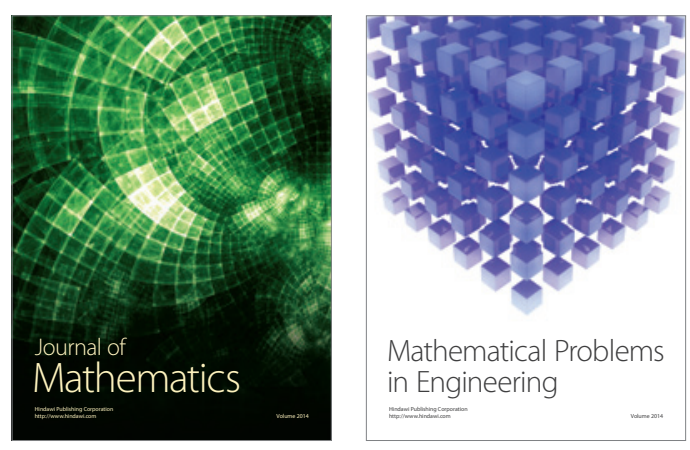

Mathematical Problems in Engineering
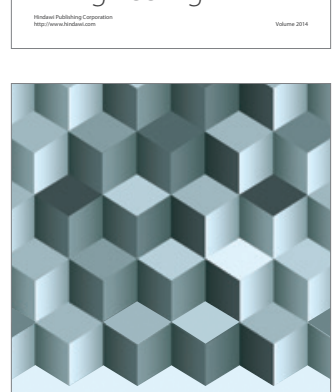

Journal of

Function Spaces
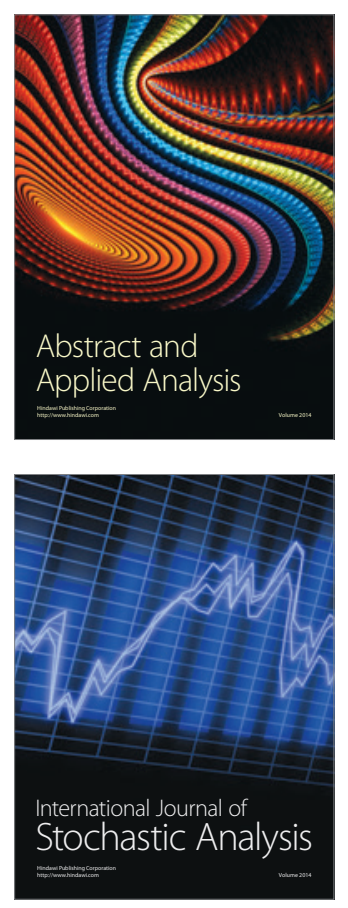

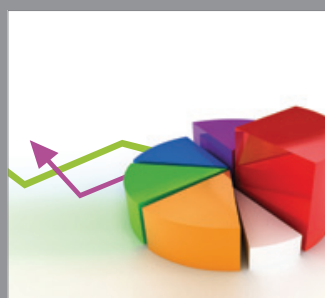

ournal of

Probability and Statistics

Promensencen
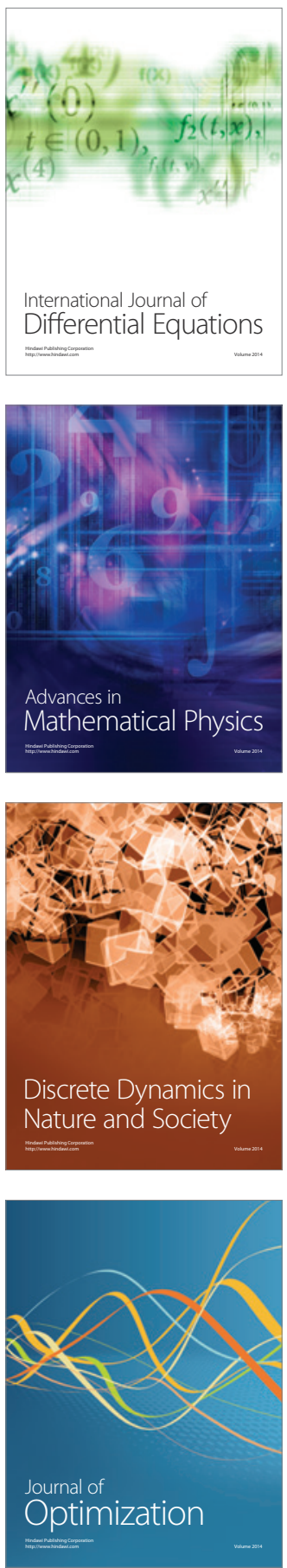